Ophthalmologe 2022 $\cdot 119: 1288-1293$

https://doi.org/10.1007/s00347-022-01591-w

Eingegangen: 2. Dezember 2021

Überarbeitet: 8. Januar 2022

Angenommen: 28. Januar 2022

Online publiziert: 3. März 2022

(c) Der/die Autor(en) 2022

\section{Fuchs-Endotheldystrophie, Katarakt und Astigmatismus}

\author{
Ein Vergleich der Korrektur des Astigmatismus mittels DMEK \\ plus simultaner vs. sequenzieller Kataraktoperation mit \\ torischer IOL-Implantation
}

\author{
Loïc Hamon (iD - Tim Berger · Berthold Seitz • Loay Daas \\ Klinik für Augenheilkunde, Universitätsklinikum des Saarlandes (UKS), Homburg/Saar, Deutschland
}

\section{Anamnese}

Eine 59-jährige Patientin stellte sich bei Fuchs-Endotheldystrophie (FECD) in unserer ambulanten Sprechstunde zur Mitbeurteilung vor. Anamnestisch gab die Patientin eine seit Monaten zunehmende Lichtempfindlichkeit, hauptsächlich vormittags, sowie eine progressive Visusminderung an beiden Augen an. Neben einer Anisohyperopie links (sphärisches Äquivalent: $+2,75 \mathrm{D}$ ) mehr als rechts (sphärisches Äquivalent: 0 D) lag ein stabiler hoher regulärer Astigmatismus beidseits vor. Aufgrund dieser Refraktionsamblyopie betrug anamnestisch der bestkorrigierte Visus am linken Auge maximal 0,5 (dezimal).

\section{Befunde}

Bei Erstvorstellung betrug der bestkorrigierte Brillenvisus (RA: $+2,25 /-4,50 / \mathrm{A}$ $170^{\circ}$; LA: $+6,25 /-6,75 / \mathrm{A}^{\circ}$ ) am rechten Auge (RA) 0,4 und 0,25 am linken Auge (LA). Es zeigte sich spaltlampenbiomikroskopisch rechts eine fortgeschrittene Hornhaut-Endothel-Epithel-Dekompensation (HEED) mit endothelialen Guttae sowie einer feinblasigen fokalen bullösen Keratopathie von 5 bis $10 \mathrm{Uhr}$ (• Abb. 1a). Links zeigte sich eine beginnende HEED mit endothelialen Guttae ohne bullöse Keratopathie (-Abb. 1b). Der restliche Vorderabschnitt war - bis auf eine Cataracta corticonuclearis incipiens - unauffällig.

In der Scheimpflug-Tomographie der Hornhaut (Pentacam HR - Oculus GmbH, Wetzlar, Deutschland) ergab sich rechts ei- ne zentrale Pachymetrie (CCT) von $785 \mu \mathrm{m}$ mit einer parazentralen Verdickung von $1000 \mu \mathrm{m}$ im Bereich der Bullae (• Abb. 2a). Links betrug die CCT $657 \mu \mathrm{m}$. Topographisch zeigte sich ein beidseitiger regulärer Astigmatismus nach der Regel in Höhe von 4,0 D (flache Achse bei $175^{\circ}$ ) rechts und 5,8 D (flache Achse bei $180^{\circ}$ ) links (- Abb. 2b). Die Abb. 3a, b stellt die Hornhautrückfläche dar.

\section{Therapie und Verlauf}

Wir führten am rechten Auge eine „Descemet membrane endothelial keratoplasty" (DMEK) mit einem Transplantatdurchmesser von 7,5 mm ohne Linsenoperation (sog. „phake DMEK") durch. Die 8,0-mmDescemetorhexis erfolgte unter kontinuierlicher Lufteingabe mittels eines „anterior chamber maintainer" (ACM). Nach Luftentfernung wurde die Endothellamelle in die Vorderkammer injiziert und mithilfe einer $20 \%$ igen SF6-Gasblase zum Anliegen gebracht [6]. Der bestkorrigierte Visus $\left(+4,00 /-5,25 / \mathrm{A} 160^{\circ}\right)$ betrug 6 Wochen postoperativ 0,6. Die CCT sank auf $534 \mu \mathrm{m}$ mit Resorption der fokalen HEED. Der korneale Astigmatismus änderte sich auf 5,1 D bei $164^{\circ}$ (- Abb. 4 und 5).

Wir führten sequenziell, 10 Wochen nach der DMEK, eine Phakoemulsifikation mit Implantation einer torischen Intraokularlinse (IOL) (RayOne Toric 610T + 19,00 D, Zyl.6,00 D @ 71 - Rayner Intraocular Lenses Limited, Worthing, UK) durch. Die Zielrefraktion betrug 0 bis $-0,5 \mathrm{D}$ mit Vollkorrektur des Astigmatismus („target induced astigmatism" $[\mathrm{TIA}]=6,00$ @ 

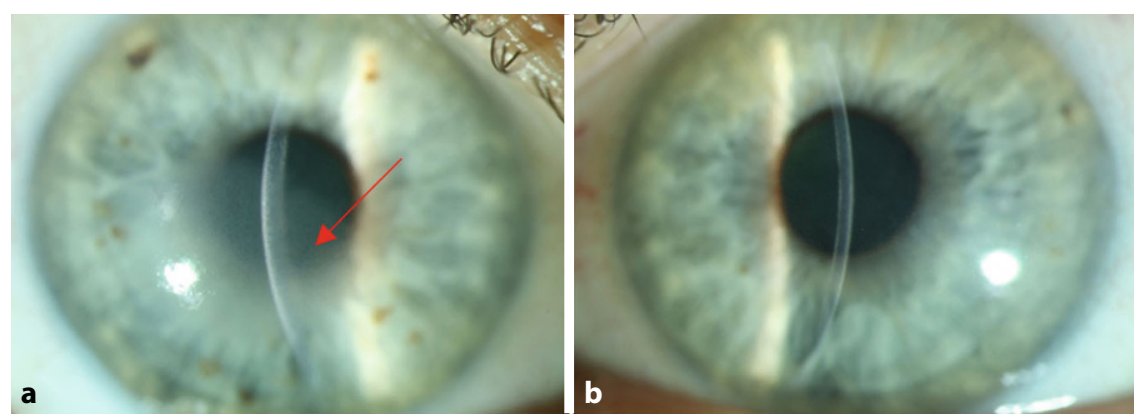

Abb. 1 ॥ Klinisches Bild. a Rechtes Auge: Fuchs-Endotheldystrophie (FECD) mit fokaler Keratopathia bullosa (Bullae nicht dargestellt) (Pfeil). b Linkes Auge: FECD ohne Keratopathia bullosa

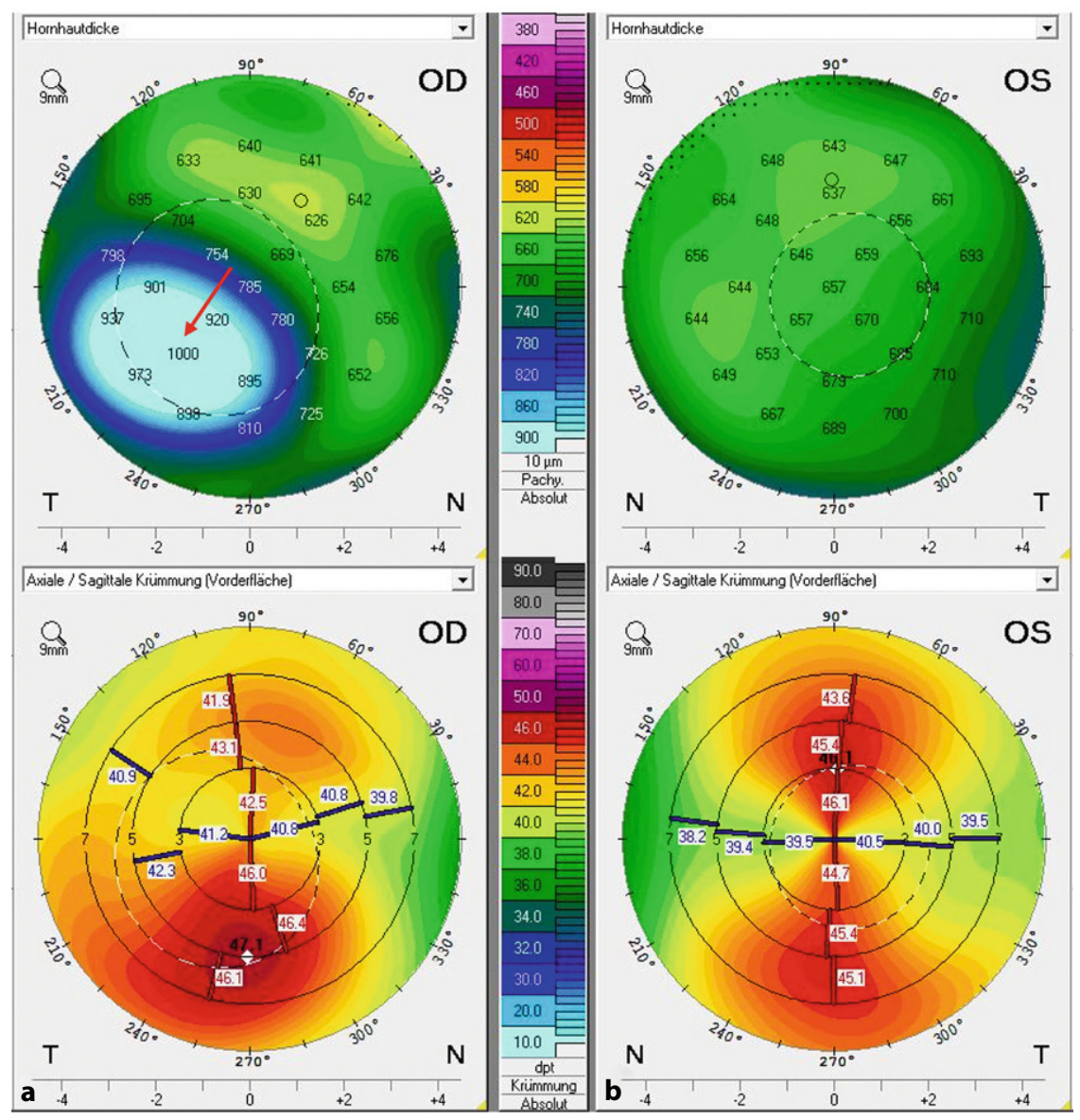

Abb. 2 \ Tomographie und simulierte Topographie der Hornhaut - Vorderfläche und Pachymetrie präoperativ. a Rechtes Auge: FECD mit schwerer fokaler mittelperipherer Verdickung bis zu $1000 \mu \mathrm{m}$ bei $8 \mathrm{~h}$ (Pfeil). b Linkes Auge: FECD mit mäßiggradiger und homogener Verdickung 


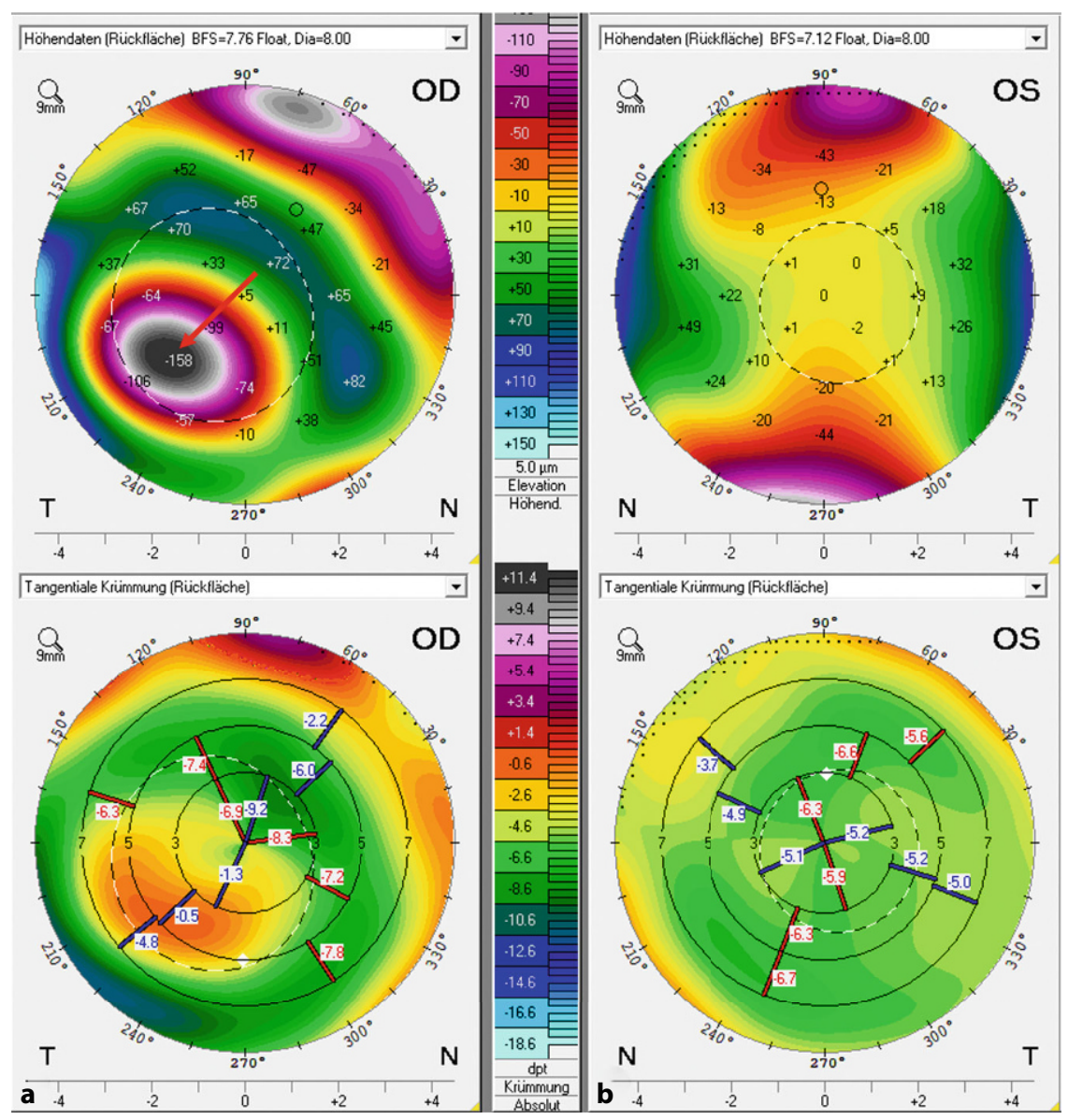

Abb. 3 ム Tomographie und simulierte Topographie der Hornhaut - Rückfläche präoperativ. a Rechtes Auge: Deutliche Inselbildung (Pfeil) im Bereich der fokalen Verdickung bei $8 \mathrm{~h}$ als Zeichen einer ödematösen Wölbung der Hornhautrückfläche in Richtung Vorderkammer auf der "Posterior-Elevation“-Karte. Auf der Rückflächenkrümmungskarte irregulärer posteriorer Astigmatismus mit fokaler Ansteilung der Rückfläche im Bereich der Bullae. b Linkes Auge: Weitgehend regulärer Befund auf den „Posterior-Elevation-“ und Rückflächenkrümmungskarten ohne fokale Wölbung $71^{\circ}$ ), ein schnittinduzierter Astigmatismus von 0,25 D@90 wurde mitkorrigiert. Postoperativ wurde ein Visus von 0,8 $\left(-0,75 /-2,50 / A \quad 105^{\circ}\right)$ nach 6 Wochen erreicht. Der "surgically induced astig-

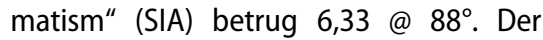
Differenzvektor (DV, entspricht SIA - TIA) betrug 3,62 @ 122 (• Abb. 6).

Wir führten 5 Wochen später eine kombinierte DMEK mit Phakoemulsifikation und Implantation einer torischen IOL (RayOne Toric 610T + 20,00 D, Zyl. 7,00 D @ 94') (sog. torische Triple-DMEK) am LA durch. Die Zielrefraktion betrug 0 bis $-0,5 \mathrm{D}$. Ein prädiktiver Fehler („prediction error" $[\mathrm{PE}]$ ) von -1,0 D wurde bei Antizipation des sog. hyperopen Shifts mitberechnet, sodass eine IOL mit zuzüglicher Korrektur von $-1,0$ D sowie mit Vollkorrektur des Astigmatismus (TIA=7,00 @ $94^{\circ}$ ) ausgewählt wurde. Ein schnittinduzierter Astigmatismus von 0,25 D @ 90 wurde darüber hinaus berechnet und mitkorrigiert. Die subjektive Refraktion war $+0,75 /-1,00 / 76^{\circ}$ mit einem bestkorrigierten Visus von 0,5 (maximal erreichbarer Visus aufgrund der Amblyopia ex anisohyperopia et astigmatismo). Die CCT nahm auf $534 \mu \mathrm{m}$ ab (- Abb. 4 und 5). Der SIA betrug 8,12 @

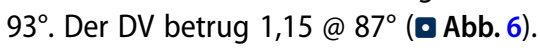

\section{Diskussion}

Die DMEK hat sich aufgrund ihrer überzeugenden klinischen Ergebnisse und niedrigen Komplikationsraten als erste Wahl zur Behandlung von endothelialen Pathologien wie der FECD oder der pseudophaken bullösen Keratopathie etabliert [6].

Torische IOLs wurden entwickelt, um einen postoperativen Hornhautastigmatismus nach Kataraktoperationen zu reduzieren und auszugleichen. Aufgrund der exzellenten visuellen Ergebnisse und der refraktiven Stabilität nach DMEK werden Berechnungen der (torischen) IOLStärke bei Patienten nach "phaker DMEK“ oder im Rahmen einer sog. Triple-DMEK in der klinischen Praxis mit großem Interesse verfolgt [2]. Voraussetzung für eine simultane torische IOL-Implantation ist eine möglichst genaue Vorhersagbarkeit der postoperativen keratorefraktiven Änderungen nach DMEK.

Bereits 2015 hat die Arbeitsgruppe um Wacker et al. die direktionalen Profilände- 


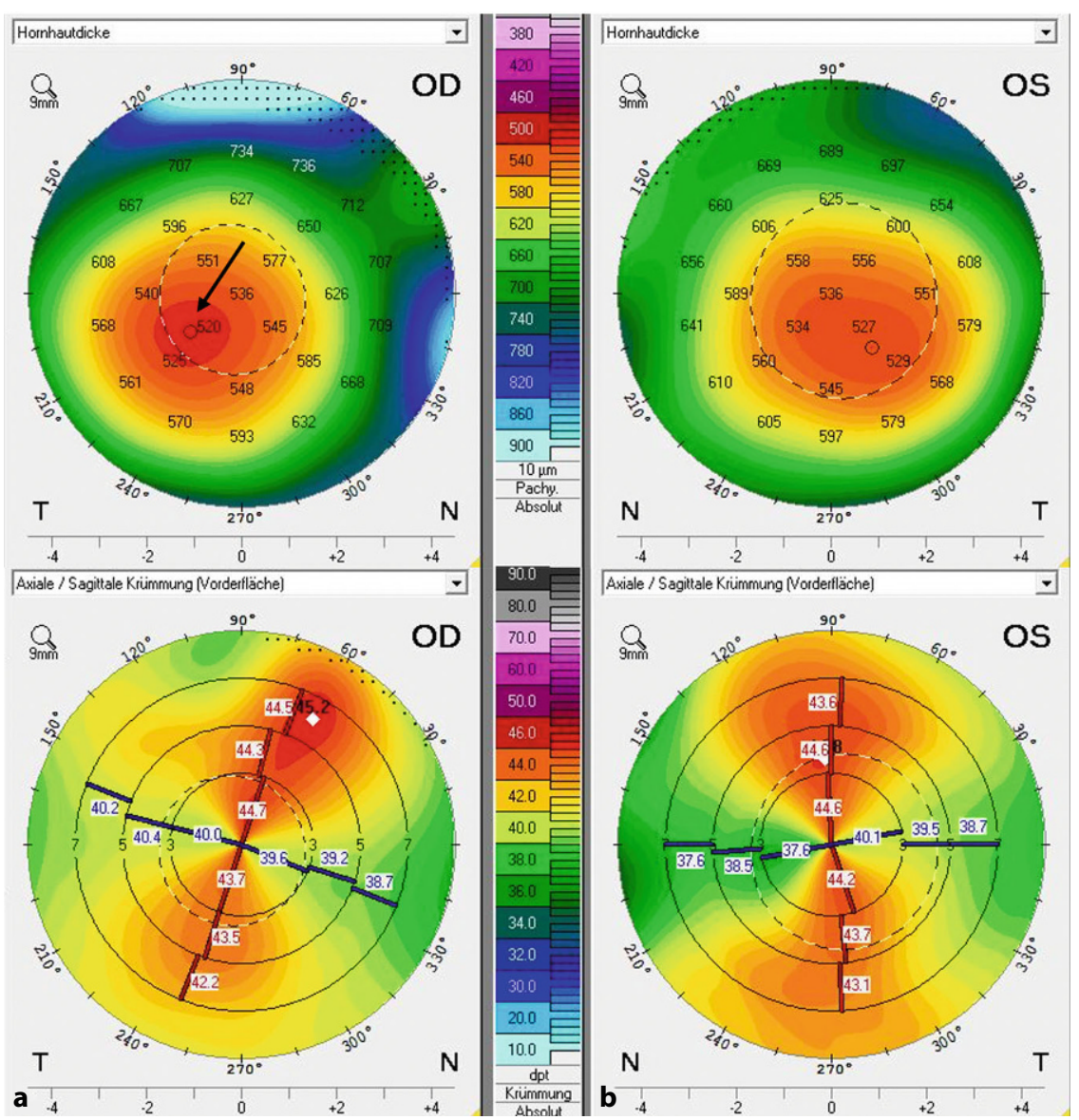

Abb. 4 \ Tomographie und simulierte Topographie der Hornhaut - Vorderfläche und Pachymetrie postoperativ. a Rechtes Auge: 16 Wochen nach phaker DMEKund 6 Wochen nach Implantation der torischen IOL mit deutlicher Resorption des kornealen Ödems, insbesondere bei $8 \mathrm{Uhr}$ (Pfeil) und eine Regularisierung des kornealen Astigmatismus. b Linkes Auge: 6 Wochen nach Triple-DMEKmit torischer IOL-Implantation rungen der Hornhautrückfläche bei FECD untersucht. Sie konnten zeigen, dass die hintere Torizität der Hornhaut sich bei fortgeschrittener FECD abnormal ändert, da die horizontale Hornhautverdickung relativ größer ist als die vertikale [7]. Diese Verdickung führt nicht selten zu einer leichten postoperativen Hyperopisierung von etwa 0,4 D (sog. hyperoper Shift) [3]. Aus diesem Grund sollte bei der Triple-DMEK mit Emmetropiewunsch einen PE von $-0,5$ bis $-1,0 \mathrm{D}$ mitgerechnet werden, damit postoperativ eine Zielrefraktion zwischen 0 und $-0,5$ D erreicht wird [3].

Die ungleiche Verdickung verursacht einen leichten Astigmatismus nach der Regel an der kornealen Rückfläche [2]. Nach einer (Triple-)DMEK ändert sich die korneale Rückfläche wieder zu einem Astigmatismus gegen die Regel wie vor dem Auftreten der FECD. Diese Änderungen des Astigmatismus sind für die Mehrheit der Patienten klinisch irrelevant (ca. 0,25 D) [2]. In diesem Sinne sollte der Astigmatismus in der steilen Achse vollkorrigiert werden. Ein Schnitt-induzierter Astigmatismus von $0,25-0,3 \mathrm{D}\left(90^{\circ}\right.$ perpendikulär vom Hauptschnitt) sollte auch bei der Berechnung der torischen IOL mitkorrigiert werden [5].

Bei unserer Patientin führten wir am rechten Auge zunächst eine DMEK aufgrund der Keratopathia bullosa mit anschließender Kataraktoperation durch. Dieser sequenzielle Eingriff ermöglichte eine genauere IOL-Berechnung durch Regularisierung und Kompensierung der Hornhaut post DMEK. Nach der phaken DMEK war eine deutliche Hyperopisierung von $+2,2 \mathrm{D}$ zu sehen. Diese kann durch das präoperativ ausgeprägte Ödem mit fokaler Prominenz an der Hornhautrückfläche (- Abb. 3) und die postoperative (para)zentrale Verdünnung der Hornhaut - ähnlich wie nach einer phototherapeutischen Keratektomie an der Vorderfläche [4] - erklärt werden. Eine mögliche Erklärung für die postoperative Zunahme des Astigmatismus rechts könnte eine Unterschätzung des primären Astigmatismus aufgrund der Irregularität der Keratometrie (durch die Bullae) und eine darauffolgende genauere Messung des regulären Astigmatismus nach DMEK und Linsenoperation sein. Am linken Auge führten wir die Operation als „torische 


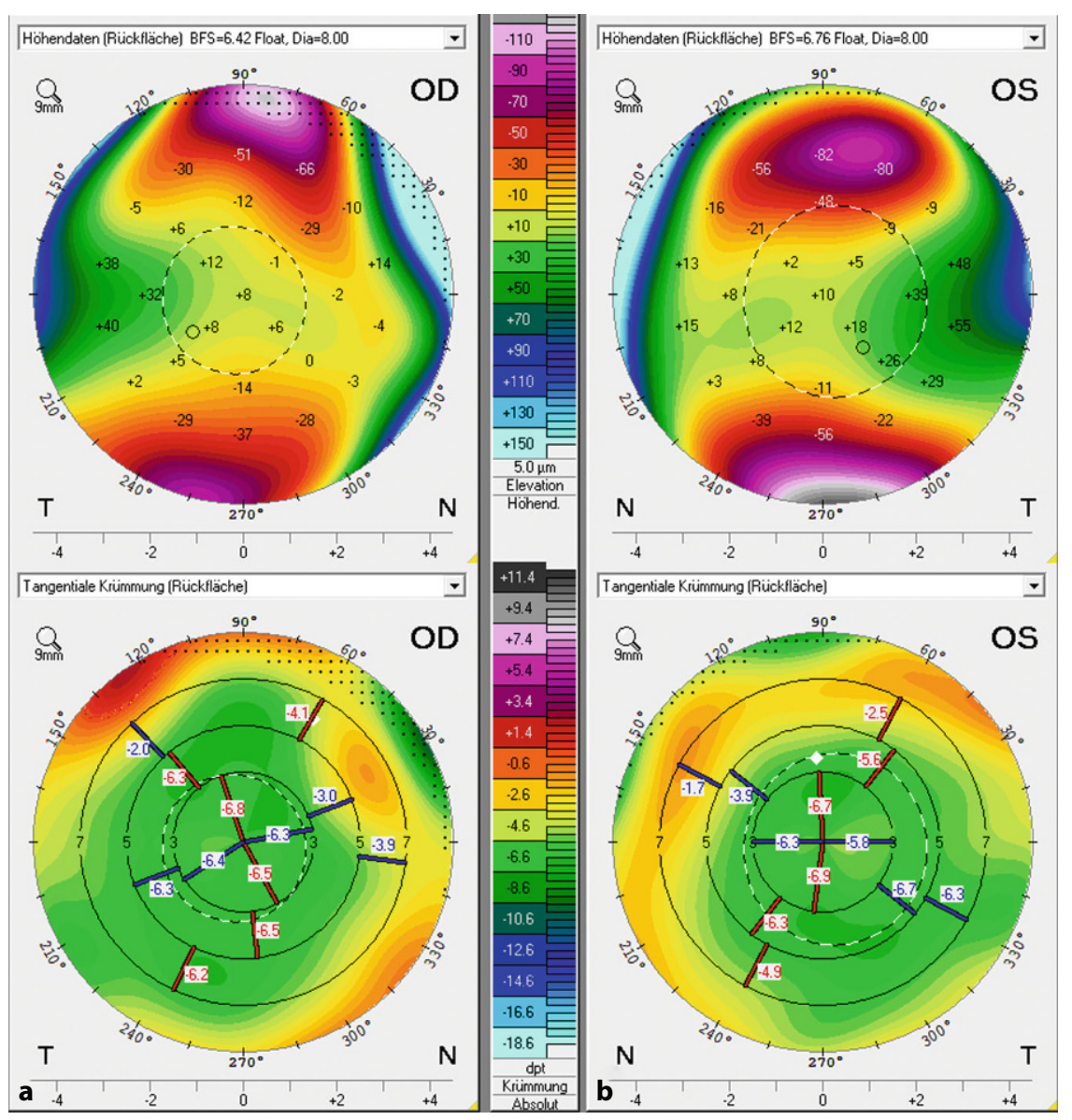

Abb. 5 \ Tomographie und simulierte Topographie der Hornhaut - Rückfläche postoperativ. a Rechtes Auge:Verschwinden der fokalen Vorwölbung nach Rückbildung des kornealen Ödems auf der „Posterior-Elevation"-Karte. Auf der Rückflächenkrümmungskarte deutliche Regularisierung des posterioren Astigmatismus. b Linkes Auge: Stabile Werte der Rückflächenkrümmung im Vergleich zu präoperativ, ohne klinisch relevante Änderung der Achsenausrichtung
Triple-DMEK" mit Antizipation des hyperopen Shifts und Mitberechnung eines PE von $-1,0$ D sowie einer Vollkorrektur des Astigmatismus durch. In beiden Fällen führten die Operationen zu einer deutlichen Emmetropisierung, jedoch links (DV=1,15@87 ) besser als rechts $\left(\mathrm{DV}=3,62\right.$ @ 122 $2^{\circ}$ (• Abb. 6).

Die torische Triple-DMEK stellt für Augen mit "einfach vorhersehbaren" refraktiven Ergebnissen, wie z.B. bei FECD, einen sicheren Eingriff dar [8]. Im Gegensatz dazu sollte bei erschwerter Linsenberechnung, wie z.B. in zuvor vitrektomierten Augen [1], in sehr kurzen Augen oder bei bullöser Keratopathie, eine sequenzielle Operationsmethode gewählt werden.

\section{Fazit für die Praxis}

- Ideale Kandidaten für eine torische TripleDMEK haben eine leichte bis mäßige HEED mit Cataracta und regulärem Astigmatismus.

- Eine fortgeschrittene FECD oder eine bullöse Keratopathie stellen aufgrund möglicher postoperativer Refraktionsabweichungen keine sichere Indikation dar.

- Bei (torischer) Triple-DMEK sollte ein prädiktiver Fehler in Richtung leichter Myopie $(-0,50$ bis $-1,00 \mathrm{D})$ aufgrund des Risikos einer Hyperopisierung bei der IOL-Auswahl mitberechnet werden, bei torischer IOLImplantation sollte zusätzlich eine Vollkorrektur des Astigmatismus angestrebt werden.

\section{Korrespondenzadresse}

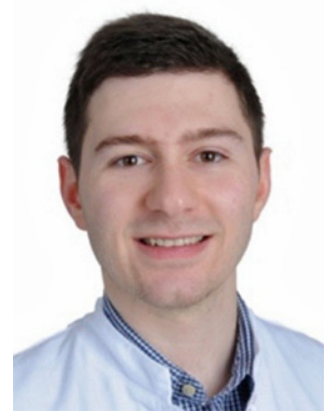

\section{Loïc Hamon}

Klinik für Augenheilkunde, Universitätsklinikum des Saarlandes (UKS)

Kirrbergerstr. 100, Gebäude 22, 66421 Homburg/Saar, Deutschland loic.hamon@uks.eu
Funding. Open Access funding enabled and organized by Projekt DEAL. 


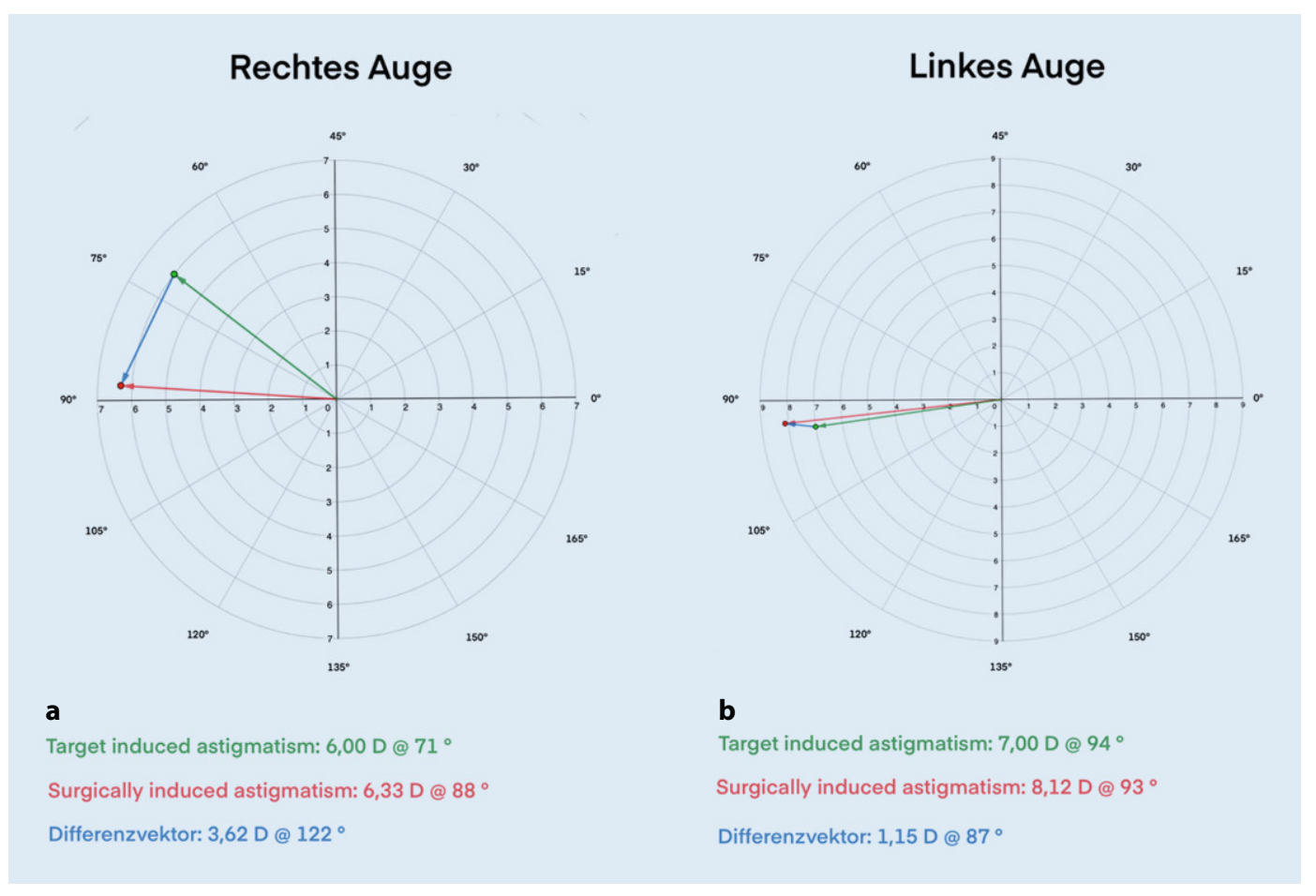

Abb. $6 \Delta$ Doppelwinkelvektoren für den Astigmatismus. a Rechtes Auge. b Linkes Auge. Der „target induced astigmatism“ (TIA) gibt die Zielkorrektur des Astigmatismus an. Der "surgically induced astigmatism“ (SIA) gibt die Änderung des Astigmatismus an, die durch den Eingriff verursacht wird. Der Differenzvektor (DV) gibt die Änderung des Astigmatismus an, die zum SIA addiert werden sollte, um die TIAzu erreichen (idealerweise $=0$ ). Am RA ist der DV trotz relativ vergleichbarer Magnitude des TIA und SIA hoch (3,62 D). Am LA ist der DV trotz größerer Diskrepanzzwischen der Magnitude des TIA und des SIA deutlich niedriger (1,15 D). Dieses Ergebnis liegt an einer größeren Abweichung der Achsenausrichtung am RA $\left(17^{\circ} \mathrm{vs} .1^{\circ}\right)$ und betont die entscheidende Rolle der adäquaten Achsenausrichtung für die astigmatische Korrektur

\section{Einhaltung ethischer Richtlinien}

Interessenkonflikt. L. Hamon, T. Berger, B. Seitz und L. Daas geben an, dass kein Interessenkonflikt besteht.

Für diesen Beitrag wurden von den Autoren keine Studien an Menschen oder Tieren durchgeführt. Für die aufgeführten Studien gelten die jeweils dort angegebenen ethischen Richtlinien. Für Bildmaterial oder anderweitige Angaben innerhalb des Manuskripts, über die Patienten zu identifizieren sind, liegt von ihnen und/oder ihren gesetzlichen Vertretern eine schriftliche Einwilligung vor.

Open Access. Dieser Artikel wird unter der Creative Commons Namensnennung 4.0 International Lizenz veröffentlicht, welche die Nutzung, Vervielfältigung, Bearbeitung, Verbreitung und Wiedergabe in jeglichem Medium und Format erlaubt, sofern Sie den/die ursprünglichen Autor(en) und die Quelle ordnungsgemäß nennen, einen Link zur Creative Commons Lizenz beifügen und angeben, ob Änderungen vorgenommen wurden.

Die in diesem Artikel enthaltenen Bilder und sonstiges Drittmaterial unterliegen ebenfalls der genannten Creative Commons Lizenz, sofern sich aus der Abbildungslegende nichts anderes ergibt. Sofern das betreffende Material nicht unter der genannten Creative Commons Lizenz steht und die betreffende Handlung nicht nach gesetzlichen Vorschriften erlaubt ist, ist für die oben aufgeführten Weiterverwendungen des Ma- terials die Einwilligung des jeweiligen Rechteinhabers einzuholen.

Weitere Details zur Lizenz entnehmen Sie bitte der Lizenzinformation auf http://creativecommons.org/ licenses/by/4.0/deed.de.

\section{Literatur}

1. Aljundi W, Abdin AD, Suffo S, Seitz B, Daas L (2021) Descemet-Membran-Endothel-Keratoplastik (DMEK) in zuvor vitrektomierten Augen: Komplikationen und klinische Ergebnisse. Klin Monatsbl Augenheilkd. https://doi.org/10.1055/ a-1517-4518

2. Alnawaiseh $M$, Zumhagen $L$, Rosentreter $A$, Eter N (2017) Changes in anterior, posterior and total corneal astigmatism after descemet membrane endothelial keratoplasty. JOphthalmol 2017:4068963

3. Fritz M, Grewing V, Böhringer D, Lapp T, Maier $P$, Reinhard T, Wacker K (2019) Avoiding hyperopic surprises after descemet membrane endothelial keratoplasty in Fuchs dystrophy eyes by assessing corneal shape. Am J Ophthalmol 197:1-6. https:// doi.org/10.1016/j.ajo.2018.08.052

4. Geerling G, Sekundo W (2006) Phototherapeutische Keratektomie (PTK). Unerwünschte Wirkungen, Komplikationen und Vermeidungsstrategien. Ophthalmologe 103:576-582
5. Langenbucher A, Viestenz A, Szentmáry N, Seitz B, ViestenzA (2008) Pseudophake und phake torische Linsen zur Korrektur des kornealen Astigmatismus - Theorie und klinische Aspekte. Klin Monbl Augenheilkd 225:541-547

6. Seitz B, Daas L, Flockerzi E, Suffo S (2020) "Descemet membrane endothelial keratoplasty" DMEK - Spender und Empfänger Schritt für Schritt. Ophthalmologe 117:811-828

7. Wacker K, McLaren JW, Patel SV (2015) Directional posterior corneal profile changes in Fuchs' endothelial corneal dystrophy. Invest Ophthalmol Vis Sci 56:5904-5911. https://doi.org/10.1167/ iovs.15-17311

8. Yokogawa H, Sanchez PJ, Mayko ZM, Straiko MD, Terry M (2017) Astigmatism correction with toric intraocular lenses in descemet membrane endothelial keratoplasty triple procedure. Cornea 36:269-274 